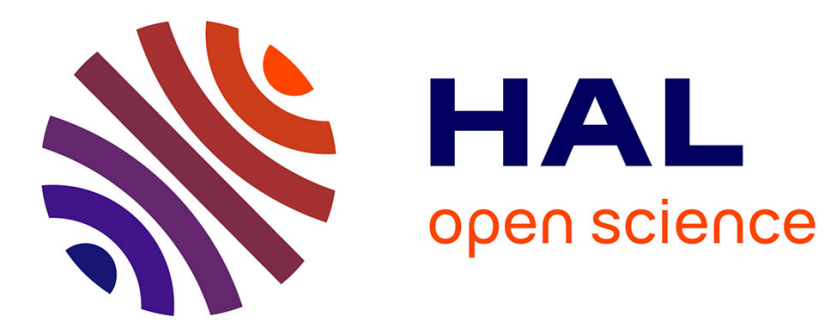

\title{
A Note on Maximal Lattice Growth in $\mathrm{SO}(1, \mathrm{n})$
}

Jean Raimbault

\section{To cite this version:}

Jean Raimbault. A Note on Maximal Lattice Growth in $\mathrm{SO}(1, \mathrm{n})$. International Mathematics Research Notices, 2013, 2013 (16), pp.3722 - 3731. 10.1093/imrn/rns151 . hal-01593685

\section{HAL Id: hal-01593685 \\ https://hal.science/hal-01593685}

Submitted on 26 Sep 2017

HAL is a multi-disciplinary open access archive for the deposit and dissemination of scientific research documents, whether they are published or not. The documents may come from teaching and research institutions in France or abroad, or from public or private research centers.
L'archive ouverte pluridisciplinaire HAL, est destinée au dépôt et à la diffusion de documents scientifiques de niveau recherche, publiés ou non, émanant des établissements d'enseignement et de recherche français ou étrangers, des laboratoires publics ou privés. 


\title{
A note on maximal lattice growth in $\mathrm{SO}(1, n)$
}

\author{
Jean Raimbault ${ }^{1}$ \\ ${ }^{1}$ Institut de Mathématiques de Jussieu, Unité Mixte de Recherche 7586 du CNRS, Université Pierre \\ et Marie Curie, 4, place Jussieu 75252 Paris Cedex 05, France. \\ Correspondence to be sent to: raimbault@math.jussieu.fr
}

We show that the number of maximal lattices with volume less than $v$ is at least exponential in $v$. To do this we construct families of noncommensurable Gromov/Piatetski-Shapiro manifolds having the same volume.

\section{Introduction}

In their famous paper [6] Gromov and Piatetski-Shapiro have shown how to construct examples of nonarithmetic hyperbolic $n$-manifolds in any dimension $n \geq 3$ by gluing together submanifolds of noncommensurable standard arithmetic manifolds. Here we aim to show that their methods have other applications, for example we construct arbitrarily large families of noncommensurable manifolds with the same volume:

Theorem 1.1. Let $n \geq 3$; there exists constants $V, K>0$ depending on $n$ such that for all integers $m \geq V$ there is a family of at least $2^{m}$ noncommensurable (nonarithmetic) compact hyperbolic $n$-manifolds all having the same volume $v \leq K m$.

This can be seen as giving an explicit lower bound in Wang's theorem [10] that there are only finitely many hyperbolic $n$-manifolds $(n \geq 4)$ with volume less than a given $v$, however in that respect it is much less good than existing results. For example, it is proven in [4] that for any large integer $m$ there are at least $m$ ! hyperbolics $n$-manifolds the same volume less than $K^{\prime} m$ (for some $K^{\prime}$ depending on the dimension $n$ ). However all these manifolds are constructed as coverings of a fixed manifold, so that our result is slightly different from theirs or those proved in [5] or [11], in particular it implies:

Corollary 1.2. Let $L_{c o m}(v)$ be the number of commensurability classes of compact hyperbolic $n$-manifolds which have an element with volume less than $v$. Then

$$
\liminf _{v \rightarrow \infty} \frac{\log L_{c o m}(v)}{v}>0 .
$$

Since there is at least one maximal subgroup with minimal covolume in each commensurability class, it also follows from Theorem 1.1 that for $v \geq V$ there exists at least $2^{\frac{v}{K}}$ maximal (nonarithmetic) uniform lattices with covolume less than $v$. Interestingly, the growth of maximal arithmetic subgroups is subexponential by a result of Misha Belolipetsky (cf. [2]), so that the growth rate of maximal nonarithmetic subgroups is strictly bigger.

Corollary 1.3. Let $L_{\max }(v)$ (resp. $L_{a, \max }(v)$ ) be the number of conjugacy classes of maximal uniform lattices in $\mathrm{SO}(1, n)$ (resp. arithmetic ones) with covolume less than $v$. Then $L_{\max }$ is at least exponential, in particular we have:

$$
\lim _{v \rightarrow \infty} \frac{\log L_{a, \max }(v)}{\log L_{\max }(v)}=0 .
$$

For $n=3$ the number of lattices with volume less than $v$ is infinite for $v$ big enough, but this number becomes finite if we add the condition that the systole be bigger than any positive constant. In our theorem the manifolds have their systoles bounded below by a positive constant. As an aside, we also construct manifolds with arbitrarily large volume the size of whose isometry group stays bounded. All our constructions are quite costless in technology (we only use the Gromov/Piatetski-Shapiro paper). 


\section{Gluing manifolds}

\subsection{General construction}

Let $r \geq 2$ and $N_{1}, \ldots, N_{r}$ be real compact hyperbolic $n$-manifolds such that each has totally geodesic boundary, and each boundary is the disjoint union of two copies of some hyperbolic $(n-1)$-manifold $\Sigma$. We choose for each manifold $N_{a}$ a component $\Sigma_{a}^{+}$of $\partial N_{a}$, and denote the other one by $\Sigma_{a}^{-}$; we call $i_{a}^{ \pm}$the corresponding embeddings of $\Sigma$ in $\partial N_{a}$. For an integer $m>0$ we identify the cyclic group $\mathbb{Z} / m \mathbb{Z}$ with the set $\{0, \ldots, m-1\}$. For $\alpha=\left(\alpha_{0}, \ldots, \alpha_{m-1}\right) \in\{1, \ldots, r\}^{\mathbb{Z} / m \mathbb{Z}}$ we define $M_{\alpha}$ to be the closed hyperbolic manifold obtained by gluing copies of the $N_{k}$ in the manner prescribed by $\alpha$ :

$$
M_{\alpha}=\left(\bigsqcup_{i \in \mathbb{Z} / m \mathbb{Z}} N_{\alpha_{i}} \times\{i\}\right) /\left\{\forall i \in \mathbb{Z} / m \mathbb{Z}, x \in \Sigma,\left(i_{\alpha_{i}}^{+} x, i\right)=\left(i_{\alpha_{i+1}}^{-} x, i+1\right)\right\} .
$$

For $k=0, \ldots, m$ we shall take the (abusive) convention of denoting by $N_{\alpha_{k}}$ the submanifold image of $N_{\alpha_{k}} \times\{k\}$ in $N_{\alpha}$ and by $\Sigma_{k}$ the hypersurface image of $i_{\alpha_{k}}^{-}(\Sigma) \times\{k\}$. In the same way, for a subsequence $\left(\alpha_{k}, \ldots, \alpha_{k+l}\right)$ we denote by $N_{\alpha_{k}, \ldots, \alpha_{k+l}}$ the submanifold of $N_{\alpha}$ image of the $N_{\alpha_{k}} \times\{k\}, \ldots, N_{\alpha_{k+l}} \times\{k+l\}$.

\subsection{Interbreeding arithmetic manifolds}

According to the construction of Proposition 4.1 below there exists a family $M_{k}, k \geq 1$ of arithmetic hyperbolic closed $n$-manifolds such that any two of them are noncommensurable but they all contain an embedded nonseparating totally geodesic hypersurface isometric to some fixed $\Sigma$. Letting $N_{k}$ be the completion of $M_{k}-\Sigma$ (so that it has boundary $\Sigma \sqcup \Sigma$ ), for all $r>0$ we can perform with $N_{1}, \ldots, N_{r}$ the constructions of Section 2.1. (Gromov and Piatetski-Shapiro call this interbreeding of manifolds, and the resulting nonarithmetic manifolds are called hybrids). There is a natural (shift) action of $\mathbb{Z} / m \mathbb{Z}$ on $\{1, \ldots, r\}^{\mathbb{Z} / m \mathbb{Z}}$ and it is clear that we obtain the same manifolds for two collections of indices in the same orbit. The main technical result is that this is the only case when they are commensurable (recall that two Riemannian manifolds are said to be commensurable if they share a finite cover up to isometry). For a sequence $\gamma \in\{1, \ldots, r\}^{\mathbb{Z} / m \mathbb{Z}}$ we denote by $\bar{\gamma}$ its symmetric sequence, i.e. $\bar{\gamma}_{j}=\gamma_{-j}$.

Proposition 2.1. Let $\alpha_{1}, \ldots, \alpha_{m}, \beta_{1}, \ldots, \beta_{m} \in \mathbb{N}$. The manifolds $M_{\alpha_{1}, \ldots, \alpha_{m}}$ and $M_{\beta_{1}, \ldots, \beta_{m}}$ are commensurable if and only if there is a p such that either $\forall j, \alpha_{j+p}=\beta_{j}$ or $\forall j, \alpha_{p-j}=\beta_{j}$, i.e. if and only if $\alpha$ and $\beta$ or $\bar{\beta}$ are in the same $\mathbb{Z} / m \mathbb{Z}$-shift orbit.

We will prove this in section 3 below. Now we give the proof of our main result.

\subsection{Manifolds with the same volume}

We keep the same notation as above and denote $\operatorname{vol}\left(M_{k}\right)=v_{k}$. Theorem 1.1 follows immediately from the case $r=2$ of the next result, which gives a more precise estimate.

Proposition 2.2. For all positive integers $r, m$ there exists a family of noncommensurable closed hyperbolic $n$-manifolds all having the same volume $\sum_{i=1}^{r} m v_{i}$ which contains exactly $a_{m}$ elements, and $a_{m} \underset{m \rightarrow \infty}{\sim}$ $(2 m)^{-1}(2 \pi m)^{-\frac{r-1}{2}} r^{r m-1}$

Proof. Let $S$ be the set of all manifolds $M_{\alpha_{1}, \ldots, \alpha_{r m}}$ such that for each $k=1, \ldots, r$ exactly $m$ of all $\alpha_{i}$ are equal to $k$, modulo commensurability. All manifolds in this set have volume $\sum_{i=1}^{r} m v_{i}$. According to Proposition 2.1 the cardinality of $S$ is bigger than $(2 \mathrm{rm})^{-1}$ times the number of such collections of $\alpha_{i}$. This last number is equal to:

$$
\left(\begin{array}{c}
m r \\
m
\end{array}\right) \times\left(\begin{array}{c}
m(r-1) \\
m
\end{array}\right) \times \ldots \times\left(\begin{array}{c}
2 m \\
m
\end{array}\right)=\frac{(r m) !}{(m !)^{r}} \sim_{m \rightarrow \infty}(2 \pi m)^{-\frac{r-1}{2}} r^{r m}
$$

where the asymptotic follows from Stirling's formula.

We get a constant $K$ proportional to the volume $\max \left(v_{1}, v_{2}\right)$, in particular it could be estimated using the explicit construction of Proposition 4.1 and Prasad's volume formula. Note that taking a bigger $r$ would not improve on the growth rate since the constant $K \gg \max \operatorname{vol}\left(v_{1}, \ldots, v_{r}\right)$ would tend to infinity too quickly (stricly faster than $\log r$, as follows from the estimates in [2]) so that the exponential growth rate $r^{1 / K}$ would tend to 1. 


\subsection{Manifolds with small group of isometries}

We take the same notation as in the preceding subsection, with $r=2$.

Proposition 2.3. There exists a family $S_{k}, k \geq 1$ of closed hyperbolic $n$-manifolds such that $\operatorname{vol}\left(S_{k}\right) \rightarrow \infty$ but the groups of isometries $\operatorname{Isom}\left(S_{k}\right)$ have uniformly bounded order.

Note that the systole of the $M_{k}$ stays bounded below by a constant. The main result of [3] together with Wang's theorem imply the much stronger result that there is a sequence of hyperbolic manifolds with volume tending to infinity and trivial group of isometries (one could perhaps obtain also a systole tending to infinity).

Proof. Let $S_{k}=M_{2,1, \ldots, 1}$ with $k$ ones, then $\operatorname{vol}\left(S_{k}\right) \geq k v_{1}$. On the other hand, an isometry of $S_{k}$ must map the $N_{2}$ piece to itself because of Lemma 3.3, so that $\left|\operatorname{Isom}\left(S_{k}\right)\right| \leq\left|\operatorname{Isom}\left(N_{2}\right)\right|$. It remains to show that the group $\operatorname{Isom}\left(N_{2}\right)$ is finite: this is because an isometry is determined by its restriction to the boundary, so that $\left|\operatorname{Isom}\left(N_{2}\right)\right| \leq 2\left|\operatorname{Isom}^{+}(\Sigma)\right|<+\infty$.

\section{Noncommensurability}

\subsection{Some lemmas}

We recall here some results that will be useful for proving the noncommensurability of the manifolds constructed in Section 2.1. The main advantage of working with arithmetic manifolds is that the following criterion for commensurability is available (see $[6,1.6]$ ).

Proposition 3.1. If $\Gamma, \Gamma^{\prime}$ are two arithmetic subgroups in $\mathrm{SO}(1, n)$ such that the intersection $\Gamma \cap \Gamma^{\prime}$ is Zariskidense in $\mathrm{SO}(1, n)$, then this intersection has finite index in both of them (so that they are in particular commensurable).

We will also require the following lemma, whose proof is similar to that of Corollary 1.7B in [6].

Lemma 3.2. Let $n \geq 3, W$ a complete hyperbolic $n$-manifold and $U, U^{\prime}$ two submanifolds with compact totally geodesic boundary. Then either the intersection $U \cap U^{\prime}$ has empty interior or it contains an hyperbolic $n$-manifold with Zariski-dense fundamental group.

Proof. We fix a monodromy for the hyperbolic structure on $W$; then for any totally geodesic submanifold we get a monodromy as a subgroup of $\pi_{1}(W) \subset \mathrm{SO}(1, n)$. Suppose that $U \cap U^{\prime}$ contains an open set and let $S$ (resp. $S^{\prime}$ ) be a component of $\partial U$ (resp. $\partial U^{\prime}$ ) which intersects the interior of $U^{\prime}$ (resp. $U$ ). Then $\pi=\pi_{1}\left(S \cap U^{\prime}\right.$ ) must be Zariski-dense in the subgroup $H \cong \mathrm{SO}(1, n-1)$ of $\mathrm{SO}(1, n)$ determined by the compact hyperbolic $n$-1-manifold $S$. Indeed, let $S_{0}$ be a connected component of $S \cap U^{\prime}$. Then the boundary $\partial S_{0}$ is a (union of) connected components of $S \cap \partial U^{\prime}$ and thus a compact hyperbolic manifold of dimension $n-2$; we choose a component $S_{1}$. Lemma 1.7.A of [6] tells us that $\pi_{1}\left(S_{0}\right)$ contains $\pi_{1}\left(S_{1}\right)$ as a subgroup of infinite index. Now a discrete subgroup of $\mathrm{SO}(1, n-1)$ containing a subgroup of infinite index which is a lattice in some closed subgroup $H_{2} \cong \mathrm{SO}(1, n-2)$ must be Zariski-dense in $\mathrm{SO}(1, n-1)$ because $H_{2}$ is a maximal closed subgroup. In the same way $\pi^{\prime}=\pi_{1}\left(S^{\prime} \cap U\right)$ is Zariski-dense in another distinct embedding $H^{\prime}$ of $\operatorname{SO}(1, n-1)$. It follows that $\left\langle\pi, \pi^{\prime}\right\rangle$ is Zariski-dense in $\mathrm{SO}(1, n)$, and since it is contained in $\pi_{1}\left(U \cap U^{\prime}\right)$ this proves the lemma.

As a consequence of these two results we get:

Lemma 3.3. Let $N_{0}, N_{1}$ be two submanifolds with totally geodesic boundary inside two noncommensurable arithmetic hyperbolic $n$-manifolds $M_{0}, M_{1}$. For any complete hyperbolic manifold $W$, finite covers $N_{0}^{\prime}, N_{1}^{\prime}$ of $N_{0}, N_{1}$ and isometric embeddings $\iota_{0}, \iota_{1}: N_{0}^{\prime}, N_{1}^{\prime} \hookrightarrow W$, we have that $\iota_{0}\left(N_{0}^{\prime}\right) \cap \iota_{1}\left(N_{1}^{\prime}\right)$ has empty interior.

Proof. Suppose that some component $N$ of $\iota_{0}\left(N_{0}^{\prime}\right) \cap \iota_{1}\left(N_{1}^{\prime}\right)$ contains an open set; then by Lemma 3.2, $\pi_{1}(N)$ is Zariski-dense in $\mathrm{SO}(1, n)$. Thus, the intersection $\pi_{1}\left(N_{0}\right) \cap \pi_{1}\left(N_{1}\right)$ contains a Zariski-dense subgroup, which contradicts Proposition 3.1 and the fact that $M_{0}, M_{1}$ are noncommensurable. 


\subsection{Conclusion}

We keep notation as in the statement of Proposition 2.1. For convenience we denote $M=M_{\alpha_{1}, \ldots, \alpha_{m}}$ and $M^{\prime}=M_{\beta_{1}, \ldots, \beta_{m}}$. Suppose that $M$ is commensurable to $M^{\prime}$, let $W$ be a common finite covering of $M$ and $M^{\prime}$ with covering maps $\pi, \pi^{\prime}$. We will use the following lemma.

Lemma 3.4. If $i \neq j$ are two indices such that $\alpha_{i} \neq \alpha_{i+1}=\ldots=\alpha_{j} \neq \alpha_{j+1}$ then any connected component of $\pi^{-1}\left(N_{\alpha_{i}, \ldots, \alpha_{j}}\right)$ is equal to a connected component of $\left(\pi^{\prime}\right)^{-1}\left(N_{\beta_{i^{\prime}}, \ldots, \beta_{j^{\prime}}}\right)$ where $j^{\prime}-i^{\prime}=j-i$ and for $l=$ $1, \ldots, j-i$ we have $\beta_{i^{\prime}+l}=\alpha_{i+l}$.

Proof. Let $l \in\{0, \ldots, m-1\}$ be any index such that $\beta_{l} \neq \alpha_{i+1}$. Then by Lemma 3.3 we have that $\left(\pi^{\prime}\right)^{-1}\left(N_{\beta_{l}}\right) \cap$ $\pi^{-1}\left(N_{\alpha_{i+1}, \ldots, \alpha_{j}}\right)$ has empty interior. It follows that for any connected component $N$ of $\pi^{-1}\left(N_{\alpha_{i+1}, \ldots, \alpha_{j}}\right)$ there are $\beta_{k+1}=\ldots=\beta_{k+l}=\alpha_{i+1}$ such that $N$ is contained in a connected component $N^{\prime}$ of $\left(\pi^{\prime}\right)^{-1}\left(N_{\beta_{k+1}, \ldots, \beta_{k+l}}\right)$. But again by Lemma 3.3 we get that $N^{\prime} \cap \pi^{-1}\left(N_{\alpha_{i}}\right)$ as well as $N^{\prime} \cap \pi^{-1}\left(N_{\alpha_{j+1}}\right)$ have empty interior, which forces $N^{\prime}=N$. All that is left to show is that $l=i-j$, which follows from showing that the classes of commensurability among the $N_{0, \ldots, 0}$ are classified by the number of zeroes (and the same thing goes of course for the $N_{1, \ldots, 1}$ as well).

This is easy: if two $N_{0, \ldots, 0}$ share a finite cover then the degrees of the covers induced on the boundaries are the same so that the covering maps to the $N_{0, \ldots, 0}$ themselves have the same degree. It follows that the $N_{0, \ldots, 0}$ have the same volume and hence are the same.

Proof of Proposition 2.1. We prove the result for $r=2$, the general case is similar but notationally more cumbersome. Thus let $\alpha, \beta \in\{0,1\}^{\mathbb{Z} / m \mathbb{Z}}$ such that the manifolds $M_{\alpha}$ and $M_{\beta}$ are commensurable. Suppose first that all $\alpha_{i}$ are equal to, say, 0 . Then $M_{\alpha}$ is a cyclic cover of $M_{0}$ and in particular it is arithmetic. It follows that $M_{\beta}$ is arithmetic too and by Gromov and Piatetski-Shapiro's argument if this is the case then all $\beta_{j}$ must all be equal to an $a \in\{0,1\}$. Finally we get that $M_{0}$ and $M_{a}$ are commensurable and it follows that we have $a=0$.

Now suppose there is an index $0 \leq i_{1} \leq m-1$ such that $\alpha_{i_{1}-1} \neq \alpha_{i_{1}}$ : we may suppose that $i_{1}=0$ and $\alpha_{0}=0$. We partition $\mathbb{Z} / m \mathbb{Z}$ into the disjoint sets $\left\{\alpha_{0}, \ldots, \alpha_{i_{2}}\right\}, \ldots,\left\{\alpha_{i_{l-1}+1}, \ldots, \alpha_{m-1}\right\}$ where we have for $k=1, \ldots, l-1$ that $\alpha_{i_{k}} \neq \alpha_{i_{k}+1}=\ldots=\alpha_{i_{k+1}} \neq \alpha_{i_{k+1}+1}$. We first eliminate the case where all $i_{k+1}-i_{k}$ are equal to a fixed integer $m^{\prime}$ for $k$ even and another $m^{\prime \prime}$ for $k$ odd (i.e. the maximal sequences of 0 in $\alpha$ have all the same length $m^{\prime}$ and those of 1 have all length $m^{\prime \prime}$ ). Indeed, if this is the case then by Lemma 3.4 the maximal sequences of 0 in $\beta$ also have length $m^{\prime}$ and those of 1 have length $m^{\prime \prime}$. Up to shift there is only one sequence in $\{0,1\}^{\mathbb{Z} / m \mathbb{Z}}$ with these properties, which finishes the proof in this case.

We now take care of the remaining cases: in the notation above we may suppose that $i_{3}-i_{2} \neq i_{l}-i_{l-1}$. Then by Lemma 3.4 we get that there exists a $p$ such that $\beta_{p}=\beta_{p+1}=\ldots=\beta_{p+i_{2}-1}=0, \beta_{p-1}=\beta_{p+i_{2}}=1$ and $\pi_{\alpha}^{-1}\left(N_{\alpha_{0}, \ldots, \alpha_{i_{2}-1}}\right)=\pi_{\beta}^{-1}\left(N_{\beta_{p}, \ldots, \beta_{p+i_{2}-1}}\right)$. Furthermore, the same lemma also implies that:

(i) either the sequence of 1 preceding $\beta_{p}$

(ii) or that following $\beta_{p+i_{2}-1}$

has length $i_{3}-i_{2}$. If we are in case (i) then by applying again Lemma 3.4 we get that preceding $\beta_{p-\left(i_{3}-i_{2}\right)}$ there is a sequence of 0 of length $i_{4}-i_{3}$. Iterating this argument will eventually yield that $\forall j, \beta_{i_{2}}+p-j=\alpha_{j}$. If we are in case (ii), the same arguments imply that $\forall j, \beta_{j+p}=\alpha_{j}$.

\section{Arithmetic manifolds with totally geodesic hypersurfaces}

The following construction is now standard and goes back at least to Millson's paper [8]. We recall it here for the reader's convenience and because for an even $n$ it is not proved in [6] that one can get noncommensurable manifolds this way.

Proposition 4.1. For any $n \geq 2$ there exists countably many closed arithmetic hyperbolic $n$-manifolds which all contain some fixed $n-1$-hyperbolic manifold $S$ as an embedded nonseparating totally geodesic hypersurface and which are noncommensurable to each other.

Proof. The standard way to construct arithmetic hyperbolic manifolds which contain totally geodesic hypersurfaces is as follows: let $F$ be a totally real number field and $q$ a quadratic form in $n+1$ variables over $F$ such that $q$ is definite positive at all real places of $F$ but one, where it has signature $(1, n)$. Then the group of integer points $\Gamma=\operatorname{SO}\left(q, \mathcal{O}_{F}\right)$ is a lattice in $\operatorname{SO}(1, n)$. If $q$ is written as $a_{1} x_{1}^{2}+\ldots+a_{n+1} x_{n+1}^{2}$ where $a_{1}, \ldots, a_{n}$ are totally positive and $a_{n+1}$ is negative at exactly one real place, then $\Gamma$ contains the subgroup $\Lambda$ associated to 
the quadratic form in $n$ variables $a_{2} x_{2}^{2}+\ldots+a_{n+1} x_{n+1}^{2}$. Define the principal congruence subgroup of level $\mathfrak{p}$, $\Gamma(\mathfrak{p})$, to be the kernel $\Gamma \cap \operatorname{ker}\left(\mathrm{SL}\left(n+1, \mathcal{O}_{F}\right) \rightarrow \mathrm{SL}\left(n+1, \mathcal{O}_{F} / \mathfrak{p}\right)\right)$. It follows from the proof of Selberg's Lemma that for $|\mathfrak{p}|$ big enough the lattice $\Gamma(\mathfrak{p})$ is torsion-free, so that the subgroup $\Lambda(\mathfrak{p})$ gives rise to an imbedding $\Lambda(\mathfrak{p}) \backslash \mathbb{H}^{n-1} \rightarrow \Gamma(\mathfrak{p}) \backslash \mathbb{H}^{n}$ and a standard argument shows that this is in fact an embedding. Moreover we can choose $\mathfrak{p}$ so that this hypersurface $S$ is non separating, that is $M-S$ is connected so that it is the interior of a compact manifold with two boundary components isometric to $S$. For all this see [8, Section 2] or [6]. Finally, note that the isometry type of $S$ depends only on $a_{2}, \ldots, a_{n+1}$ and $\mathfrak{p}$.

The simplest exemple of the previous procedure is when $F=\mathbb{Q}$ and $a_{1}, \ldots, a_{n}>0, a_{n+1}<0$ but then the manifolds obtained are noncompact for $n \geq 4$. However, if $F=\mathbb{Q}(\sqrt{d})$ for a square-free rational integer $d>1$, $a_{1}, \ldots, a_{n} \in \mathbb{Q}_{+}^{*}$ and $a_{n+1} / \sqrt{d} \in \mathbb{Q}^{*}$ then $q$ is anisotropic over $F$ so that $\Gamma_{q} \backslash \mathbb{H}^{3}$ is compact. In the sequel we make the simplest choice $d=2, a_{1}=\ldots=a_{n}=1$ and $a_{n+1}=-\sqrt{2}$. Put $q_{a}=a x_{1}^{2}+x_{2}^{2}+\ldots+x_{N}^{2}-\sqrt{2} x_{n+1}^{2}$. We want to show that there is an infinite number of $a \in \mathcal{O}_{F}$ such that the lattices $S O\left(q_{a}, \mathcal{O}_{F}\right)$ are all noncommensurable to each other. This boils down, by $[6,2.6]$, to show that the quadratic forms $q_{a}, \lambda q_{a^{\prime}}$ are nonisometric for all $a, a^{\prime}$ and $\lambda \in F^{*}$. Suppose first that $n$ is odd: then the discriminant of $\lambda q_{a}$ equals that of $q_{a}$ for all $\lambda$, thus we need only find a countable set $A \subset \mathcal{O}_{F}$ such that for all $a \neq a^{\prime} \in A$ we have $a / a^{\prime} \notin\left(F^{*}\right)^{2}$. This is doable since the image of $\mathcal{O}_{F}-\{0\}$ in $F^{*} /\left(F^{*}\right)^{2}$ is easily seen to be infinite.

Now we treat the case of an even $n$ : for that purpose we need to recall some definitions. Let $k$ be any field; for $u, v \in k^{*}$ the Hilbert symbol $(u, v)_{k}$ is defined in [9, III,1.1] as 1 if $1=u v^{2}+v y^{2}$ for some $x, y \in k$ and -1 otherwise. Then it is shown in [9, IV, Théorème 2] that

$$
\epsilon_{k}(q)=\prod_{i<j}\left(a_{i}, a_{j}\right)_{k}
$$

is an isometry invariant of $q$ over $k$. Now we suppose that $k=\mathbb{Q}_{p}$ for a prime $p>2$, then for $a, b \in \mathbb{Z}_{p}$ we have $(a, b)_{\mathbb{Q}_{p}}=-1$ if and only if either $a$ (resp. $b$ ) has odd $p$-valuation and $b$ (resp. $a$ ) has even valuation but is not a square in $\mathbb{Q}_{p}$, or if $a, b$ have the same $p$-valuation $\bmod 2$ and $-a^{-1} b$ is a square in $\mathbb{Q}_{p}$ (see $[9$, III, Théorème 2$]$ ).

Now suppose that we have a rational prime $p$ such that $p=-1(\bmod 8)$. Then 2 is a biquadratic residue modulo $p$ according to Gauss' biquadratic reciprocity law, and since $p \neq 1(\bmod 4)$ we know that -1 is not a square modulo $p$. By Hensel's lemma it follows that all this is still true in $\mathbb{Q}_{p}$. For any $\lambda \in \mathbb{Q}_{p}^{*}$ it follows that $(\lambda, \lambda)_{\mathbb{Q}_{p}}=1,(\lambda,-\sqrt{2} \lambda)_{\mathbb{Q}_{p}}=-1$ and more generally, for any $u \in \mathbb{Z}_{p}^{*},(\lambda u,-\sqrt{2} \lambda)_{\mathbb{Q}_{p}}=-(\lambda u, \lambda)_{\mathbb{Q}_{p}}$ We can now compute:

$$
\begin{aligned}
\epsilon_{\mathbb{Q}_{p}}\left(\lambda q_{u}\right) & =(\lambda u, \lambda)_{\mathbb{Q}_{p}}^{n-1} \times(\lambda,-\sqrt{2} \lambda)_{\mathbb{Q}_{p}}^{n-1} \times(\lambda u, \lambda \sqrt{2})_{\mathbb{Q}_{p}} \\
& =-(\lambda u, \lambda)_{\mathbb{Q}_{p}} \times(\lambda,-\sqrt{2} \lambda)_{\mathbb{Q}_{p}}=1 .
\end{aligned}
$$

Now if we let $p_{k}, k \geq 1$ be an infinite increasing sequence of primes all equal to -1 modulo 8 (such a sequence exists by Dirichlet's theorem) we get by (4.1) that for all $l<k$ we have $\epsilon_{\mathbb{Q}_{p_{k}}}\left(q_{p_{l}}\right)=1$. On the other hand, we have $\epsilon_{\mathbb{Q}_{p_{k}}}\left(q_{p_{k}}\right)=\left(p_{k},-\sqrt{2}\right)_{\mathbb{Q}_{p_{k}}}=-1$. It follows that for $l<k$ and $\lambda \in F \subset \mathbb{Q}_{p_{k}}$ the quadratic forms $q_{p_{k}}$ and $\lambda q_{p_{l}}$ are not isometric over $F$, so that the lattices $\mathrm{SO}\left(q_{p_{k}}, \mathcal{O}_{F}\right)$ are noncommensurable one to the other.

\section{Remarks}

1. Our construction also produces families of nonuniform noncommensurables lattices if we take the manifolds used as bulding blocks to be noncompact.

2. Instead of gluing along circles one could glue along regular graphs of higher valence. For example, it is known that in the construction of Proposition 4.1 one can actually take a cover where the hypersurface has two distinct preimage such that their union is nonseparating [7]. One can then glue their complements along a 4 -valent graph. Since there are about $C v^{v} 4$-valent graphs having $v$ vertices, if one could show that we get noncommensurable manifolds for different graphs (and appropriate colourings of the vertices) one would get the lower bound $\log L_{c o m}(v) \geq c v \log v$, which is of the order of the lower bound for the number of hyperbolic manifolds of volume less than $v$ given in [4]. However it seems harder to show noncommensurability for different graphs than for different colourings of the same graphs (for example all 4-valent graphs are commensurable).

\section{Acknowledgments}

Part of this work comes from the joint work [1] (see also the announcement in CRAS Tome 349, Fascicule 15-16) where it was used for other purposes. I thank my co-authors for valuable discussions on the contents of this note. I am also grateful for useful comments from the particiants in the Geometric Group Theory seminar in Orsay, especially Frederic Haglund and Thomas Haettel, for their many pertinent remarks during a talk I gave there. 


\section{References}

[1] M. Abert, N. Bergeron, I. Biringer, T. Gelander, N. Nikolov, J. Raimbault, and I. Samet. On the growth of $L^{2}$ invariants for sequences of lattices in Lie groups. In preparation.

[2] Mikhail Belolipetsky. Counting maximal arithmetic subgroups. Duke Math. J., 140(1):1-33, 2007. With an appendix by Jordan Ellenberg and Akshay Venkatesh.

[3] Mikhail Belolipetsky and Alexander Lubotzky. Finite groups and hyperbolic manifolds. Invent. Math., 162(3):459-472, 2005.

[4] M. Burger, T. Gelander, A. Lubotzky, and S. Mozes. Counting hyperbolic manifolds. Geom. Funct. Anal., 12(6):1161-1173, 2002.

[5] Vincent Emery. Arbitrarily large families of spaces with the same volume. Geometriae dedicata, 156(1), 2012.

[6] M. Gromov and I. Piatetski-Shapiro. Nonarithmetic groups in Lobachevsky spaces. Inst. Hautes Études Sci. Publ. Math., (66):93-103, 1988.

[7] Alexander Lubotzky. Free quotients and the first Betti number of some hyperbolic manifolds. Transform. Groups, 1(1-2):71-82, 1996.

[8] John J. Millson. On the first Betti number of a constant negatively curved manifold. Ann. of Math. (2), 104(2):235-247, 1976.

[9] Jean-Pierre Serre. Cours d'arithmétique. Presses Universitaires de France, Paris, 1977. Deuxième édition revue et corrigée, Le Mathématicien, No. 2.

[10] Hsien Chung Wang. Topics on totally discontinuous groups. In Symmetric spaces (Short Courses, Washington Univ., St. Louis, Mo., 1969-1970), pages 459-487. Pure and Appl. Math., Vol. 8. Dekker, New York, 1972.

[11] B. Zimmermann. A note on hyperbolic 3-manifolds of the same volume. Monatsh. Math., 117(1-2):139-143, 1994. 